\title{
Ectopic thymoma presenting as a giant intrathoracic tumor: A case report
}

\author{
Masahiro Kitada ${ }^{1 *}$, Kazuhiro Sato ${ }^{1}$, Yoshinari Matsuda ${ }^{1}$, Satoshi Hayashi ${ }^{1}$, Yoshihiko Tokusashi $^{2}$, Naoyuki Miyokawa ${ }^{2}$ \\ and Tadahiro Sasajima ${ }^{1}$
}

\begin{abstract}
Ectopic thymoma rarely presents as an intrathoracic tumor. We report a case of ectopic thymoma presenting as a giant right intrathoracic tumor that was treated with resection. The patient was a 50-year-old Japanese woman who presented with the chief complaint of chest pain. Detailed examination revealed a solid tumor measuring 15 $\times 10 \times 8 \mathrm{~cm}$ in diameter, with a clear border. The Imaging findings suggested a solitary fibrous tumor, and surgery was performed. At surgery, the tumor was found to beadherent to the diaphragm, mediastinal pleura, and lower lobe of the lung, although it could be dissected with relative ease and was removed. Pathological diagnosis indicated a type B1 tumor with no capsular invasion according to the World Health Organization classification, and a diagnosis of Masaoka stage I thymoma was made. No continuity with the normal thymus tissue was seen, and the thymoma was considered to be derived from ectopic thymic tissue in the pleura.
\end{abstract}

\section{Background}

Thymomas usually manifest in the anterior-superior mediastinum, and ectopic thymomas account for only $4 \%$ of all thymomas. Among ectopic thymoma, intrathoracic tumors of pleural origin are rather rare. We report, herein, a patient with a giant intrathoracic tumor that was discovered during a clinical workup to determine the cause of chest pain in the patient. The tumor was diagnosed as a thymoma that was difficult to differentiate from solitary fibrous tumor (SFT) by diagnostic imaging.

\section{Case}

A 50-year-old Japanese woman with the chief complaint of chest pain was examined at a local hospital. A chest radiograph revealed a giant tumor in the right lower lung field, and the patient was referred to our department. The patient had no pertinent personal or family history, and had never smoked. Respiratory sounds in the right lower lung field were diminished, but no other abnormalities were detected on physical examination. Respiratory function tests revealed a vital capacity (VC) of 1,470 mL and a percent predicted $\mathrm{VC}$ of $46.7 \%$, indicative of restrictive pulmonary disease. No abnormalities were

\footnotetext{
*Correspondence: k1111@asahikawa-med.ac.jp

'Department of Surgery, Asahikawa Medical University, Midorigaoka-Higashi

2-1-1-1, Asahikawa, Hokkaido 078-8510, Japan

Full list of author information is available at the end of the article
}

identified on blood biochemistry. A plaine chest $\mathrm{x}$-ray (Figure 1) showed a giant tumor shadow measuring $15 \times$ $13 \mathrm{~cm}$ in the right lower lung field, and chest computed tomography (Figure 2) showed a solid tumor measuring $15 \times 10 \times 8 \mathrm{~cm}$ in the right thoracic cavity. The tumor showed a clear borders and internal calcification, and was found todisplace the diaphragm downward and the heart to the left. In addition, on chest magnetic resonance imaging (MRI) (Figure 3), the tumor was visualized as an isointensity relative to the skeletal muscle on T1-weighted images, while on T2-weighted images, partial inclusion of weak signals hypointensity of moderate signal strength. Moreover, diffusion-weighted imaging revealed slightly heterogeneous signal hyperintensity, but no findings suggestive of degeneration or necrosis. A fibrous septum was found within the tumor, which showed a trabecular growth pattern, which led to the diagnosis of SFT, and surgery was performed. A small, the sixth intercostal video-assisted thoracotomy was performed, and the tumor was found to be slightly adherent to the diaphragm, mediastinal pleura, and lower lobe of the right lung. However, the tumor could be relatively easily dissected from these organs and removed. No continuity with normal thymic tissue was seen. Intraoperative rapid pathological diagnosis indicated a thymoma or lymphoma, and the surgery was terminated. The excised specimen (Figure 4) showed a tumor measuring $5 \times 10 \times 8 \mathrm{~cm}$ and weighing $430 \mathrm{~g}$, 

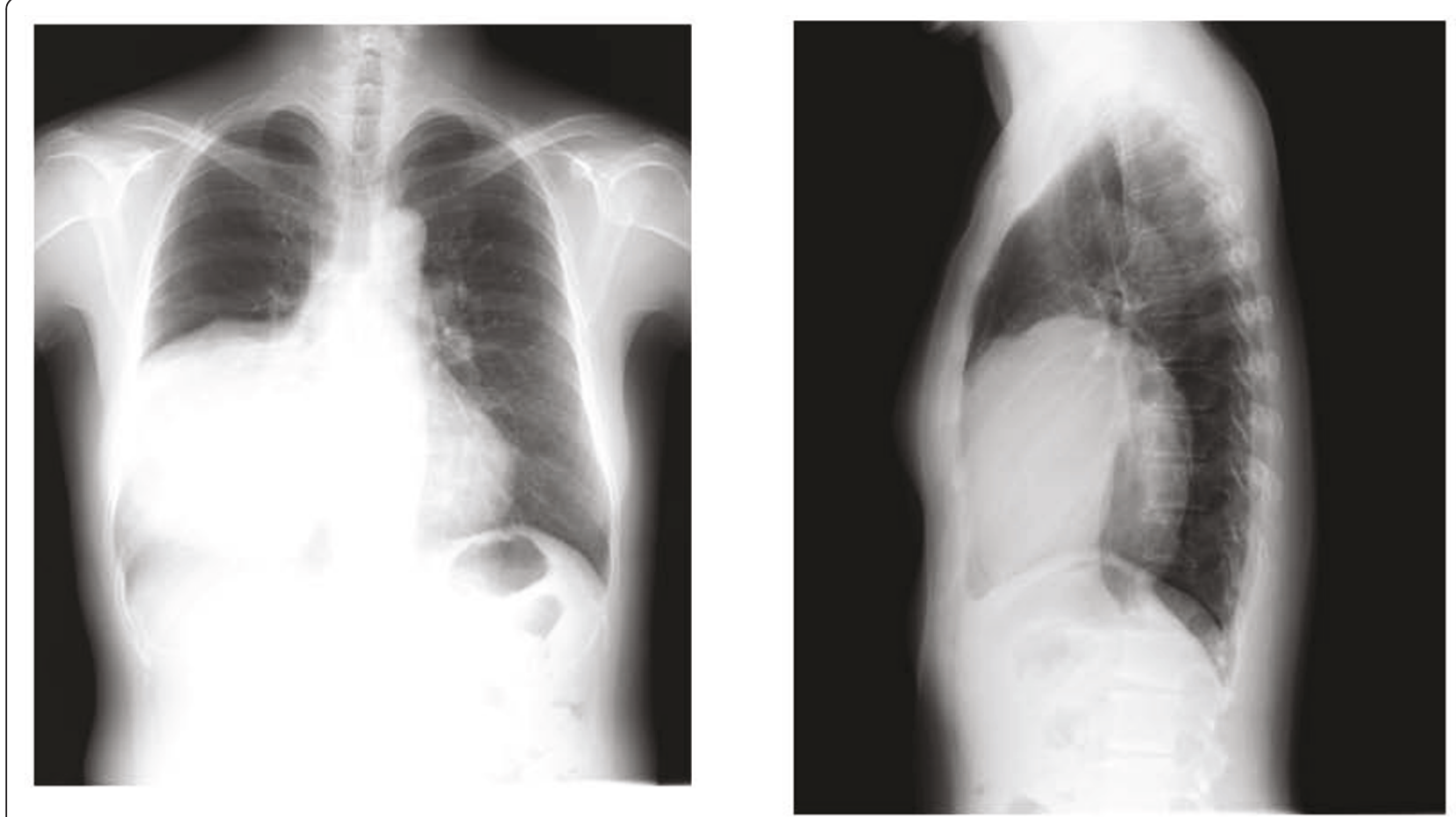

Figure 1 Plain chest radiograph showing a mass lesion in the right lower lung.

covered by a thin, fibrous membrane. The mass was elastic and soft, and the cut surface was lobulated and pale brown in color. Histopathological examination of sections stained

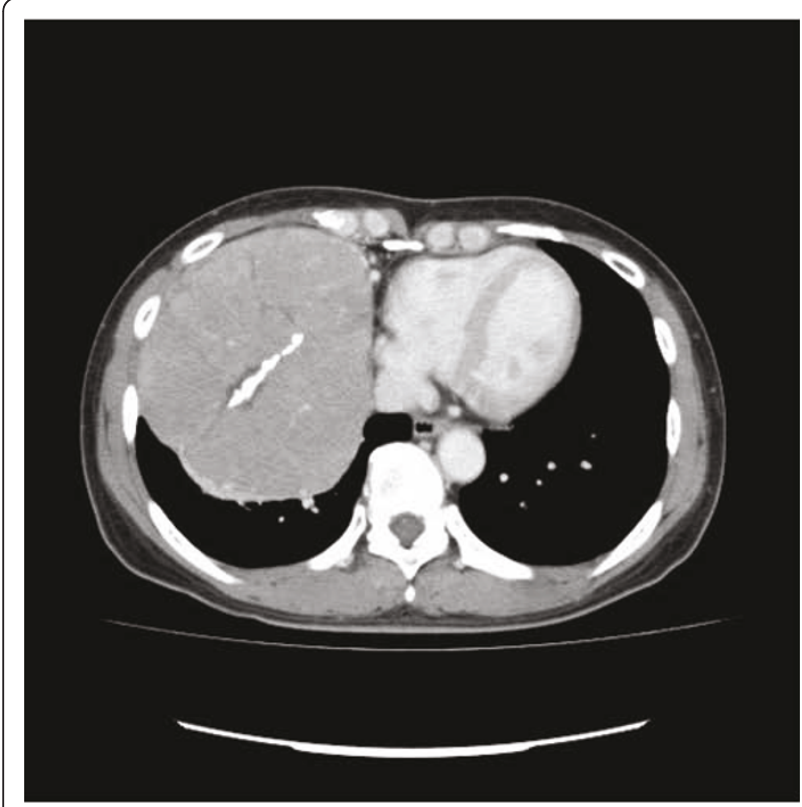

Figure 2 Chest computed tomograph showing a solid tumor $(15 \times 10 \times 8 \mathrm{~cm})$ with a clear borders and internal calcification in the right thoracic cavity. with hematoxylin-eosin (HE) (Figure 5) revealed abundant lymphocytes and large, bright tumor cells. No invasion of the capsule was evident, leading to the diagnosis of a Masaoka stage I thymoma. Immunohistochemical staining (Figure 6) showed mature lymphocytes mainly composed of $\mathrm{T}$ cells, mixed in a complex pattern with cytokeratinpositive epithelial cells. These findings led to the diagnosis of lymphocyte-predominant thymoma (type B1 thymoma). The postoperative course was good, and the patient has shown no evidence of recurrence as at the time of writing.

\section{Discussion}

Thymomas are tumors developing mainly in the thymus, are located in the anterior mediastinum, with $96 \%$ of the tumors occurring in the anterior or anterosuperior mediastinum, and only $4 \%$ being ectopic tumors [1,2]. Ectopic thymomas have been described in the neck [3], middle mediastinum $[4,5]$ posterior mediastinum, lung [6], and pleura $[7,8]$, few reports have described giant intrathoracic tumors. Thymomas are generally asymptomatic, but symptoms such as chest pain and respiratory discomfort can be caused by compression of the surrounding organs due to growth of the tumor. Symptoms such as superior vena cava syndrome can also be coused by tumor invasion of the surrounding tissues, myasthenia gravis, pure red cell apalasia, hypogammaglobulinemia. These symptoms/complications can lead to the discovery of the tumor. In addition, some patients show 

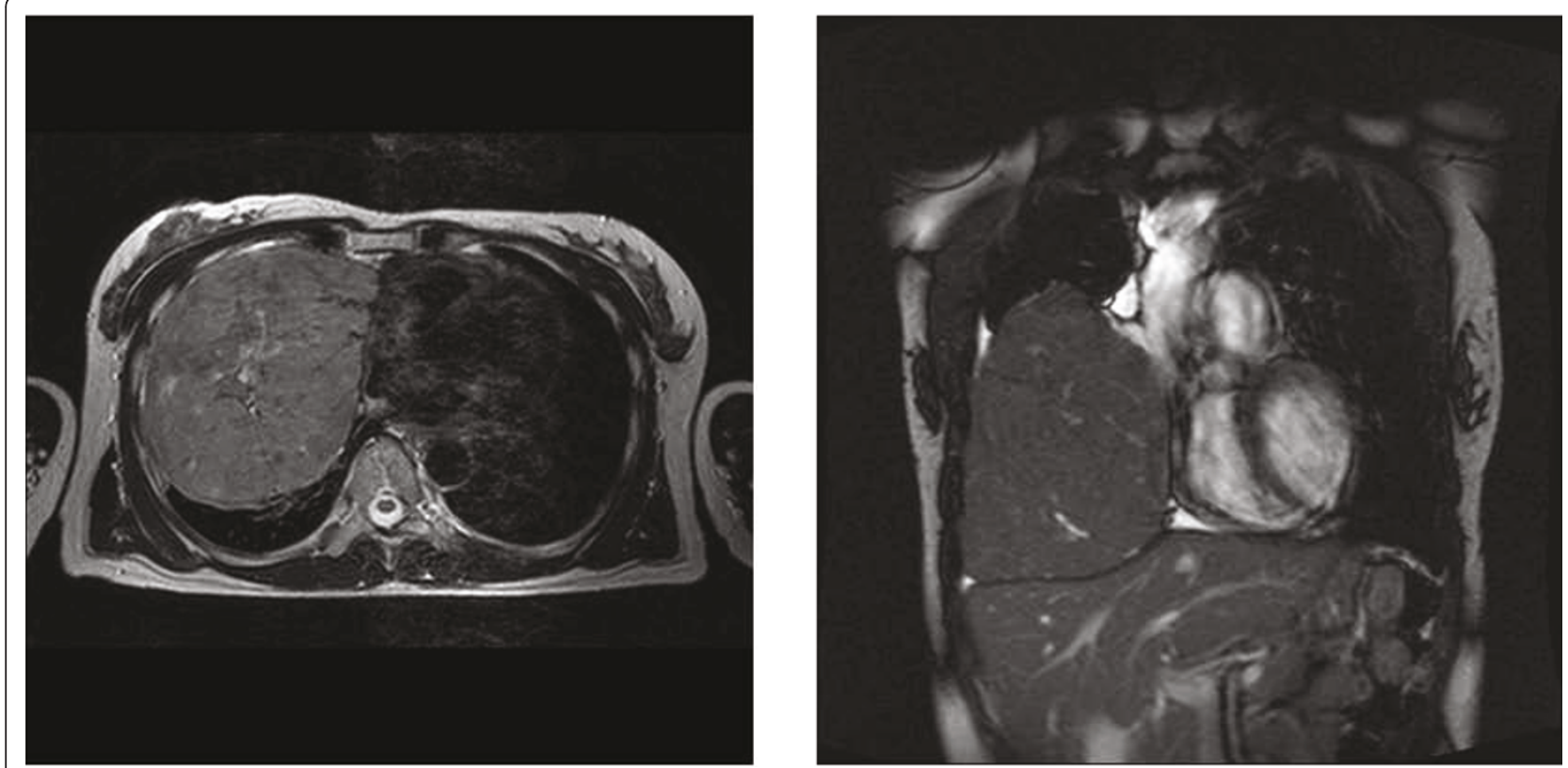

Figure 3 Chest magnetic resonance imaging. The tumor appeared isointense relative to the skeletal muscle on T1-weighted image, while T2weighted images show partial inclusion of weak signal hypointensities of moderate signal strength.

multiple lung metastases or pleural dissemination arising from recurrence or metastasis. The intrathoracic tumor in the present patient was discovered during the course of a clinical workup for chest pain, caused by compression of the surrounding organs. The patient also showed restricted impairment of pulmonary function due to the pressure on normal lung tissue, and surgical removal of the thymoma as quickly as possible was therefore considered necessary.

Definitive diagnosis is needed before surgical removal of a thymoma is planned. The differential diagnosed for giant intrathoracic tumors include SFTs, tumors of pleural origin, such as malignant pleural mesothelioma

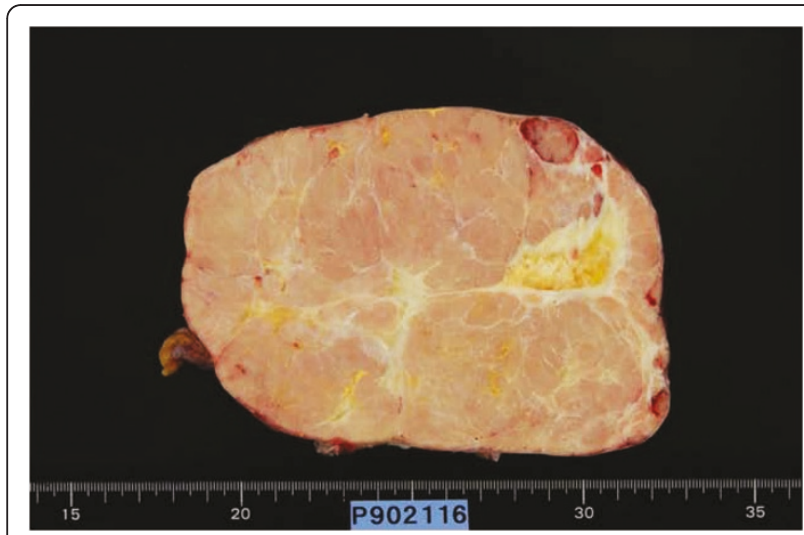

Figure 4 Excised specimen of the tumor showing a smooth margin and calcification within. or sarcoma, chest wall tumors, and metastatic tumors. In our patient, SFT was initially suspected on the basis of the MRI findings, including the shape, signal status under various weightings, and the presence of numerous linear non-signals that were considered to indicate flow voids within the lesion. It was considered that percutaneous needle biopsy would yield a definitive diagnosis, but this procedure was not performed considering the risk of tumor cell dissemination and bleeding from the tumor. Hemorrhagic shock induced by spontaneous rupture of a giant thymoma has been reported [9], and caution is warranted when considering biopsy.

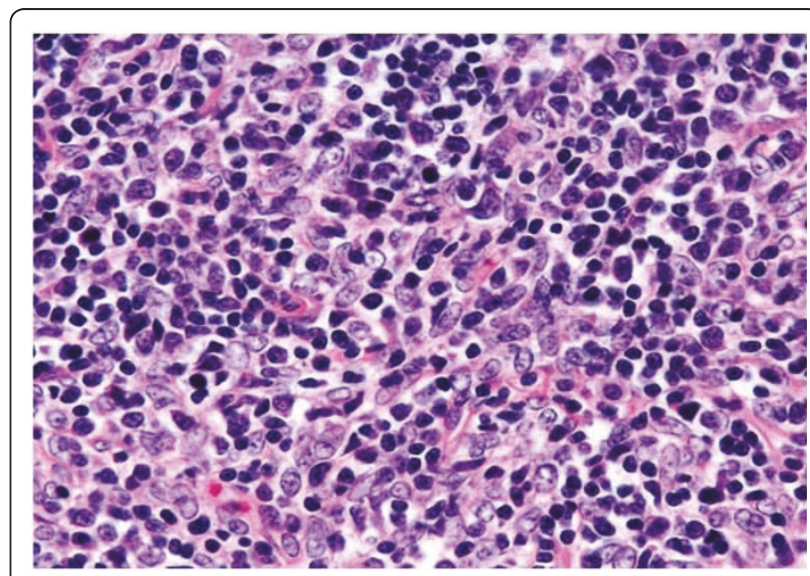

Figure 5 Histopathological examination (hematoxylin and eosin, $\times 400$ ) revealed abundant lymphocytes and large, bright tumor cells. Cells with chromatin-poor nuclei are evident. 


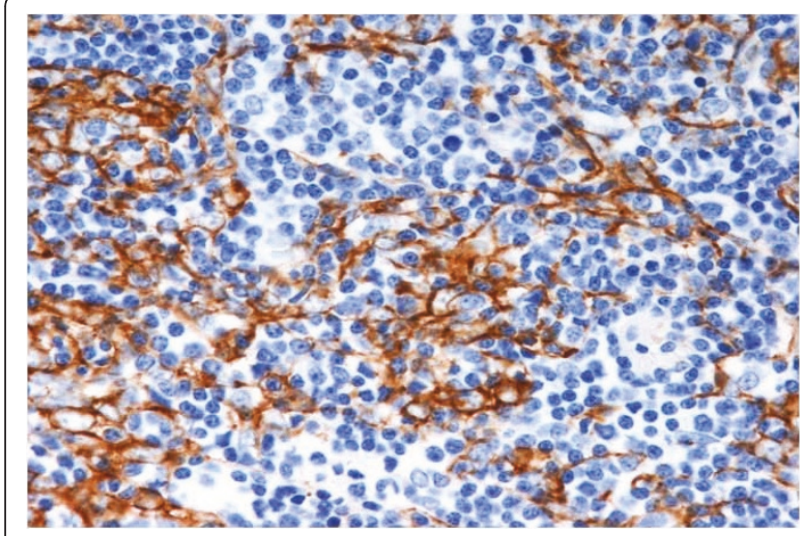

Figure 6 Immunohistochemical staining (keratin staining $\times$ 400) showing epithelial cells distributed in a mesh-like form, mixed among lymphocytes at a ratio of nearly 1:1.

\section{Consent statement}

Informed consent was obtained from the patient for publication of this case report and of the accompanying images. A copy of the written consent is available for review by the Editor-in-Chief of this journal.

\section{Author details}

${ }^{1}$ Department of Surgery, Asahikawa Medical University, Midorigaoka-Higashi 2-1-1-1, Asahikawa, Hokkaido 078-8510, Japan. ${ }^{2}$ Department of Clinical Pathology, Asahikawa Medical University, Midorigaoka-Higashi 2-1-1-1, Asahikawa, Hokkaido 078-8510, Japan.

\section{Authors' contributions}

MK operated on this case and analyzed all the data. KO, KS, YM, and SH assisted in the operation. YT and NM diagnosed the pathology of this case. TS did a professor of the Department of Surgery and had the guide of this paper. All authors read and approved the final manuscript.

\section{Competing interests}

The authors declare that they have no competing interests.

Received: 27 February 2011 Accepted: 28 June 2011

Published: 28 June 2011

\section{References}

1. Detterbeck FC, Parsons AM: Thymic tumors. Ann Thorac Surg 2004, 77:1860-69.

2. Fukayama M, Maeda Y, Funata N, Koike M, Saito K, Sakai T, Ikeda T: Pulumonary and pleural thymoma. Diagnostic application of lymphocyte markers to the thymoma of unusual site. Am J Clin Pathol 1998, 89:617-21

3. Yan B, Lim D, Petersson F: Ectopic cervical thymoma: a report of two cases of a rare entity frequently misdiagnosed on fine needle aspiration cytology and frozen section. Head Neck Pathol 2010, 4:152-6.

4. Nakamura Hiroshige, Adachi Yoshi, Fukuoka Shinji, Miwa Ken, Haruki Tomohiro, Taniguchi Yuji: Thoracoscopic Resection of middle mediastinal noninvasive thymoma; report of a case. Surg Today 2007, 37:787-789.

5. Minniti Salvatore, Valentini Marvi, Pinali Lucia, Malago Roberto, Lestani Maurizio, Procacci Carlo: Thymic mass of the middle mediastinumReport of 2 cases and review of the literature. J Thoracic Imaging 2004, 19:192-195

6. Moran $C A$, Suster $S$, Fishback $N F$, et al: Primary intrapulmonary thymoma. A clinicopathologic and immunohistochemical study of eight cases. Am J surg Pathol 1995, 19:304-12.
7. Moran CA, Travis WD, Rosado-de Christenson M, Koss MN, Rosai J: Thymomas presenting as pleural tumors: report of eight cases. Am J Surg Pathol 1992, 16:138-44.

8. Yamazaki K, Yoshino I, Oba T, Yohena T, Kameyama T, Tagawa T, Kawano D, Koso H, Maehara Y: Ectopic pleural thymoma presenting as a giant mass in the thoracic cavity. Ann Thorac Surg 2007, 83:315-17.

9. Santoprete Stefano, Ragusa Mark, Urbani Moira, Puma Francesco: Shock induced by spontaneous rupture of a giant thymoma. Ann Thorac Surg 2007, 83:1526-28.

doi:10.1186/1477-7819-9-66

Cite this article as: Kitada et al:: Ectopic thymoma presenting as a giant intrathoracic tumor: A case report. World Journal of Surgical Oncology 2011 9:66.

\section{Submit your next manuscript to BioMed Central and take full advantage of:}

- Convenient online submission

- Thorough peer review

- No space constraints or color figure charges

- Immediate publication on acceptance

- Inclusion in PubMed, CAS, Scopus and Google Scholar

- Research which is freely available for redistribution
C Biomed Central 